\title{
Hysteretic Bit/Rock Interaction Model to Analyze the Torsional Dynamics of a Drill String
}

\author{
Real F.F. ${ }^{\mathrm{a}, \mathrm{c}, \mathrm{d}}$, Batou A. ${ }^{\mathrm{b}}$, Ritto T.G. ${ }^{\mathrm{d}, *}$, Desceliers C. ${ }^{\mathrm{c}}$, Aguiar R.R. ${ }^{\mathrm{e}}$ \\ ${ }^{a}$ National Institute of Metrology, Quality and Technology-INMETRO, Rua Santa \\ Alexandrina, 416, Rio de Janeiro, RJ, 20261-232, Brazil \\ ${ }^{b}$ Department of Mechanical, Materials and Aerospace Engineering, School of \\ Engineering, University of Liverpool, Liverpool, L69 7ZF, United Kingdom \\ ${ }^{c}$ Université Paris-Est, Laboratoire Modélisation et Simulation Multi Echelle, MSME \\ UMR 8208 CNRS, 5 bd Descartes, 77454, Marne-la-Vallée, France \\ ${ }^{d}$ Department of Mechanical Engineering - Federal University of Rio de Janeiro, Ilha do \\ Fundão, Rio de Janeiro, RJ, 21945-970, Brazil \\ ${ }^{e}$ Brazil Research and Geoengineering Center, Schlumberger Oilfield Services, Rio de \\ Janeiro, Brazil
}

\begin{abstract}
The present paper proposes a novel hysteretic (non-reversible) bit/rock interaction model for the torsional dynamics of a drill string. Non-reversible means that the torque-on-bit depends not only on the bit speed, but also on the bit acceleration, producing a type of hysteretic cycle. The continuous drill string system is discretized by means of the finite element method and a reduced-order model is constructed using the normal modes of the associated conservative system. The parameters of the proposed hysteretic bit/rock interaction model is fitted with field data. The non-linear torsional vibration and the stability map of the drill string system are analyzed employing the proposed bit/rock interaction model and also a commonly used reversible model (without hysteresis). It turns out that the hysteretic model affects the stability region of the system.
\end{abstract}

Keywords: drill string nonlinear dynamics, bit/rock interaction model, torsional vibrations, stick-slip oscillations, stability map, hysteresis

\footnotetext{
*Corresponding author

Email address: tritto@mecanica.ufrj.br (Ritto T.G.)
} 


\section{Introduction}

There are several papers available in the literature concerned with the drill string torsional dynamics and stick-slip oscillations [4, 8, 5, 17, 18, 7, 23]. A pure torsional model is sometimes enough to represent this kind of system. For instance, torsional models were applied successfully to represent test rigs that were constructed in [7] to analyze the friction-induced limit cycling, and in [5] to employ a control strategy.

There are many articles available in the literature concerned with friction laws, see for instance $[24,25]$. The bit/rock interaction is very complex, but, in some situations, the relationship between the torque and the bit speed looks similar to a friction law. For instance, field measurements show (1) a torque weakening effect, and (2) hysteretic cycles [9, 19]. Nevertheless, it should be emphasized that the bit/rock interaction is a much more complex mechanism.

Field data of a five kilometer drill string is analyzed in [18], where again a pure torsional model presented satisfactory results reproducing field data, where torsional vibration was the dominant phenomenon observed. More generally, a coupled axial-lateral-torsional model should be applied [21, 17]. A full description model, including all dynamics, although possible, presents many difficulties due to lack of downhole data. During the drilling process there are many phenomena which are hard to measure, or simply not fully measured. Examples of these phenomenon include bit/rock interaction, fluid/rock interaction, proper well profile (inclination and azimuth), pipe/rock interaction, among others.

Figure 1 shows the field data [18] that will support the proposed model presented in this paper. The downhole information used in this paper was acquired using a downhole mechanics measurement unit capable of providing both real-time measurement through mud telemetry and continuously recorded high-frequency data throughout the run. The sub, installed at the BHA above the bit, contains a suite of 19 sensors sampled at 10,000 $\mathrm{Hz}$ and downsampled and filtered prior to recording at $50 \mathrm{~Hz}$. Following is a list of $50-\mathrm{Hz}$ data recorded in this sub: triaxial accelerations; gyro rpm; magnetometer rpm; axial loading; torque; bending moment [20].

The data show the dependence of the torque-on-bit with the bit speed; see Fig. 1. Closed to zero speed, the torque varies from about $6 \mathrm{kNm}$ to $11 \mathrm{kNm}$, in a very steep straight line (close to 90 degrees). As the speed increases, the torque decreases. This type of torque-on-bit and bit speed 
relation (weakening effect) has been observed experimentally before [9, 19].

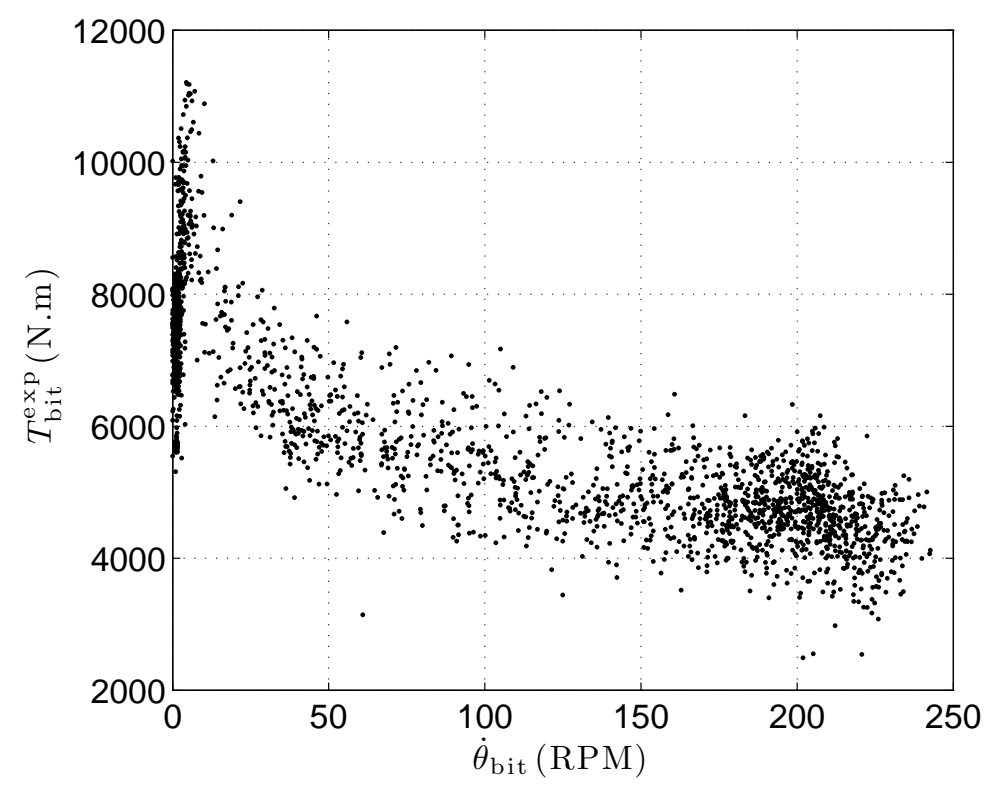

(a)

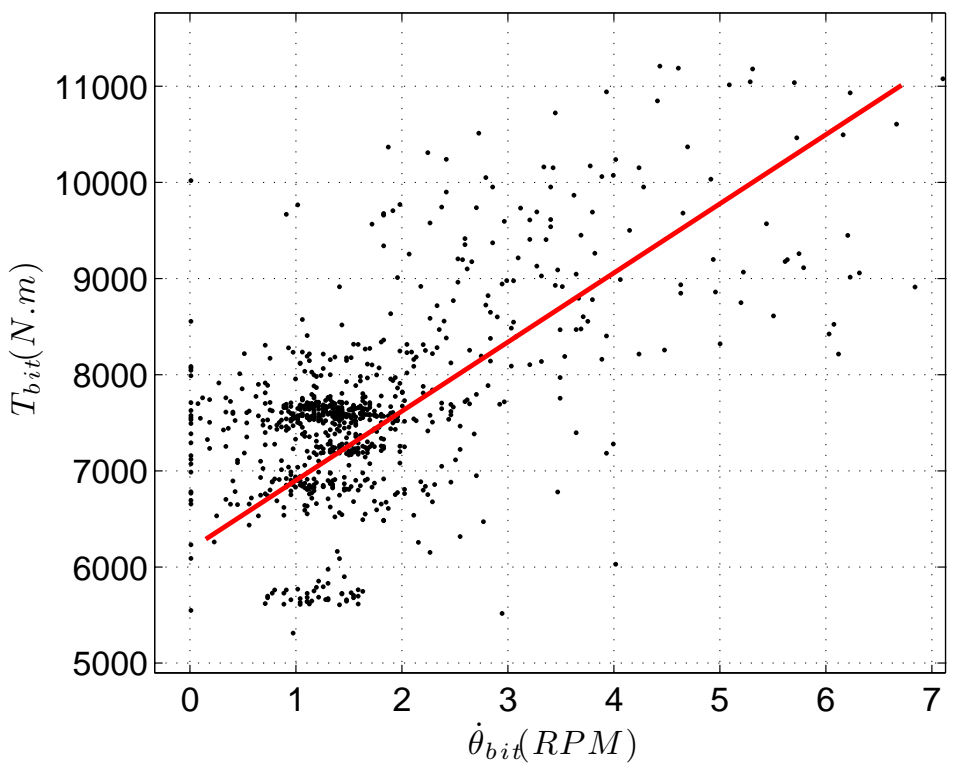

(b)

Figure 1: Field data: (a) measured torque at the BHA, very close to the drill bit $T_{\text {bit }}^{\exp }$, versus speed of the bit $\dot{\theta}_{\mathrm{bit}}$, and (b) zoom image. 
Some authors consider a non-linear function for the torque-on-bit versus bit speed to represent the bit rock/interaction model $[4,17]$, while others apply a switching mechanism [6]. One can find coupled axial-torsional bit/rock interaction models, such as [12, 22].

Hysteretic cycles have been observed experimentally for the bit/rock interaction in $[9,6]$. Up to the authors knowledge the only hysteretic bit/rock interaction model found in the literature was proposed in [1]. The authors in [1] used the experimental results presented in [6], and applied their hysteretic model, which employs a switching mechanism, in the analysis of Proportional-Integral (PI) control strategy, aiming at mitigating stick-slip oscillations.

In the present paper a novel hysteretic (non-reversible) bit/rock interaction model is proposed based on the field data presented in [18]. Nonreversible means that the torque-on-bit depends not only on the bit speed, but also on the bit acceleration, producing a type of hysteretic cycle. We call it hysteresis, even though when unloaded the torque goes back to zero. We depict the available field data, fit the model parameters with them, and argue that a hysteretic model would be appropriate for the bit/rock interaction. Then, the torsional vibration and stability map are analyzed.

This article is organized as follows. In Section 2 the torsional dynamical model is presented. The continuous system is discretized by means of the finite element method and a reduced-order model is constructed using the normal modes of the associated conservative system. The proposed bit/rock interaction model including hysteresis is also presented in Section 2. In Section 3 this interaction model is compared with field data. The numerical analysis are presented in Section 4 and, finally, the concluding remarks are made in Section 5.

\section{Dynamic Model}

\subsection{Torsional model}

The drill string system is basically composed by drill pipes (DP) and the bottomhole-assembly (BHA), as it is schematically represented in Fig. 2. $\mathrm{DP}$ are slender tubes that can reach kilometers, while BHA is composed by thicker tubes (drill collars) together with the measurement/formation logging equipment and a drill bit on the bottom, and its length usually reaches hundreds of meters. 


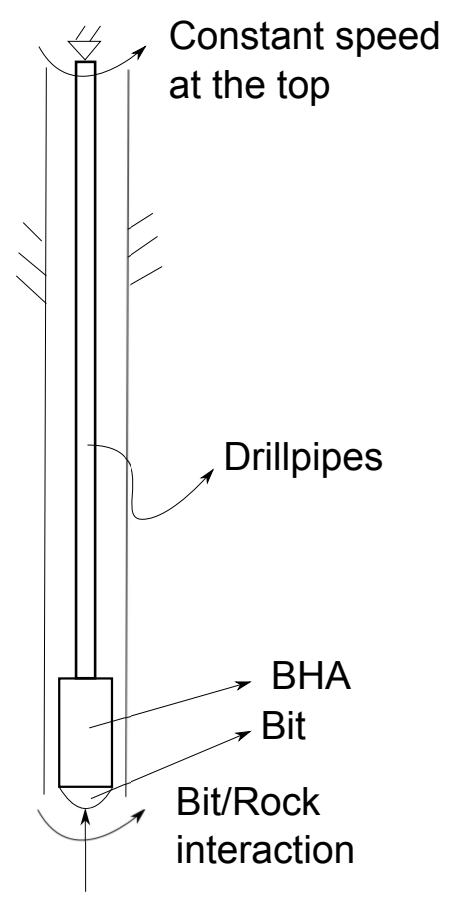

Figure 2: General scheme of a drill string system. 
A vertical wellbore is considered, and only torsional vibrations are taken into account in the modelling. In this investigation, it is assumed that the reaction forces generated during the contact between the column and the wellbore and the column with the casing are small. It is also assumed that lateral and axial vibrations are small, compared to torsional dynamics. A constant speed $\Omega$ is imposed at the top, and a reaction torque appears due to the bit/rock interaction. In the field, a control strategy, which tries to maintain the speed constant, is applied to the top drive. A PID-control law is commonly used, but there are different torsional control strategies found in the literature [10]. If the control is able to keep an almost constant speed at the top, our modeling is representative.

The angular rotation about the longitudinal axis, $\theta(x, t)$, where $x$ is the longitudinal coordinate, is the solution of the following equation of motion:

$$
\rho I_{p}(x) \frac{\partial^{2} \theta(x, t)}{\partial t^{2}}-G \frac{\partial}{\partial x}\left(I_{p}(x) \frac{\partial \theta(x, t)}{\partial x}\right)=\mathcal{T}(x, t),
$$

where, $I_{p}$ is the cross sectional polar moment of inertia, $\rho$ and $G$ are the density and shear modulus of the material of the column, and $\mathcal{T}$ is the torque per unit length. The boundary conditions at the top (constant speed) are given by:

$$
\left\{\begin{array}{c}
\theta(0, t)=\Omega t \\
\dot{\theta}(0, t)=\Omega
\end{array}\right.
$$

and the initial conditions are given by

$$
\theta(x, 0)=0, \quad \dot{\theta}(x, 0)=\Omega,
$$

Unlike $[13,15]$, the present strategy solves the system considering its rotational displacements about a rotating frame. Let $\theta^{\text {rel }}(x, t)$ be the relative angular rotation in the rotating frame associated with the imposed angle at the top. We then have

$$
\theta(x, t)=\Omega t+\theta^{\mathrm{rel}}(x, t) .
$$

The equation of motion is discretized by means of the finite element method, where linear shape functions are employed [2]. Let $\mathbf{u}(t)$ be the 
vector of nodal coordinates related to the discretization of the of the relative angular rotation $\theta^{\text {rel }}(x, t)$. Then the final discretized system, including a proportional damping model, is given by

$$
[M] \ddot{\mathbf{u}}(t)+[D] \dot{\mathbf{u}}(t)+[K] \mathbf{u}(t)=\mathbf{T}(\dot{\mathbf{u}}(t), \ddot{\mathbf{u}}(t)),
$$

where $[M]$ is the mass matrix, $[D]$ is the damping matrix, $[K]$ is the stiffness matrix, and $\mathbf{T}(\dot{\mathbf{u}}(t), \ddot{\mathbf{u}}(t))$ is the torque vector.

The initial conditions are

$$
\mathbf{u}(0)=\mathbf{0}, \quad \dot{\mathbf{u}}(0)=-\Omega \mathbf{1} .
$$

where $\mathbf{1}$ is a vector with all entries equal to one. All the components of the generalized torque vector are zero except the one corresponding to the drill bit (last node, $x=L$ ). The non-linear torque applied to the bit is denoted by $T_{\mathrm{bit}}\left(\dot{\theta}_{\mathrm{bit}}(t), \ddot{\theta}_{\mathrm{bit}}(t)\right)$ and will be described in the next section.

The normal modes of the conservative homogeneous system are used to construct a reduced-order model, considering the boundary conditions fixed at the top and free at the bottom. Few modes of the reduced-order basis give good approximations for the system analyzed, if the nonlinearity is weak. More modes are needed if the nonlinearity is severe. Other strategies, such as Karhunen-Loève Decomposition, or Proper Orthogonal Decomposition (POD) [3, 14], might be used to construct reduced-order basis for nonlinear systems.

The $m$ first eigenvalues $0<\lambda_{1} \leq \lambda_{2} \leq \ldots \leq \lambda_{m}$ associated with elastic modes $\left\{\boldsymbol{\varphi}_{1}, \boldsymbol{\varphi}_{2}, \ldots, \boldsymbol{\varphi}_{m}\right\}$ are solutions of the generalized eigenvalue problem

$$
[K] \boldsymbol{\varphi}=\lambda[M] \varphi .
$$

The reduced-order model is obtained by projecting the full computational model on the subspace spanned by the $m$ first elastic modes calculated using Eq. (7). Let $[\Phi]$ be $n \times m$ matrix whose columns are the $m$ first elastic modes. We can then introduce the following approximation

$$
\mathbf{u}(t)=[\Phi] \mathbf{q}(t)
$$

in which $\mathbf{q}(t)$ is the vector of the $m$ generalized coordinates which are solution of the reduced matrix equation

$$
[\widetilde{M}] \ddot{\mathbf{q}}(t)+[\widetilde{D}] \dot{\mathbf{q}}(t)+[\widetilde{K}] \mathbf{q}(t)=\tilde{\mathbf{T}}(\dot{\mathbf{q}}(t), \ddot{\mathbf{q}}(t))
$$


with the initial conditions

$$
\mathbf{q}(0)=\mathbf{0}, \quad \dot{\mathbf{q}}(0)=-\Omega[\widetilde{M}]^{-1}[\Phi]^{T}[M] \mathbf{1} .
$$

In these equations, $[\widetilde{M}]=[\Phi]^{T}[M][\Phi],[\widetilde{D}]=[\Phi]^{T}[D][\Phi]$ and $[\widetilde{K}]=[\Phi]^{T}[K][\Phi]$ are the $m \times m$ mass, damping and stiffness reduced-order matrices, and $\tilde{\mathbf{T}}(\dot{\mathbf{q}}(t), \ddot{\mathbf{q}}(t))=[\Phi]^{T} \mathbf{T}([\Phi] \dot{\mathbf{q}}(t),[\Phi] \ddot{\mathbf{q}}(t))$ is the reduced-order torque vector. The set of equations (8), (9) and (10) can be solved using commonly used integration schemes, such as the Euler scheme or the Runge-Kutta scheme, for instance.

Damping ratios $\zeta_{i}$ are selected directly to each i-th mode in the reduced damping matrix, which is diagonal and has entries $2 \zeta_{i} \omega_{n i}$, where $i=1, . ., n$, and $\omega_{n i}$ is the i-th natural frequency of the system. In the simulations, $\zeta_{1}=0.095, \zeta_{2,3}=0.030$, and $\zeta_{i}=0.020$ for $i>3$.

\subsection{Bit/Rock interaction model}

The field data used in this study corresponds to the drilling operation of an ultradeepwater well. Due to a research collaboration between service company and operator, the BHA was equipped with a high-frequency measurement device. The sub is capable of measuring axial forces, torque, bending moments, angular speed, and tri-axial accelerations. The data set used in this study corresponds to the drilling of about 3 meters, where only the on-bottom data (actual drilling) was used.

The field data shown in Figure 1 have been smoothed (using a time-sliding window average) to remove the measurement noise and split into stick-slip cycles. Figure 3 shows six field data stick-slip cycles. As indicate the arrows of the first graphic of Fig. 3, when the bit accelerates, the path from above occurs, and when the bit speed decreases, the path from the bottom occurs. There is a fluctuation of the hysteretic cycles. Based on field experience, the possible cause of the variability of the cycles is due to the heterogeneity of the rock formation drilled.

These cycles indicate that (1) for each cycle, the torque value for positive acceleration and for negative acceleration are not the same, this is the hysteretic (non-reversible) phenomenon and (2) each observed cycle is different from one another. The present paper tackles the first point, i.e., it proposes a bit/rock interaction model including hysteresis and representing the mean bit/rock interaction. The construction of a stochastic bit/rock interaction 
model that takes into account the fluctuation of the hysteretic cycle is out of the scope of this paper and will be pursued in a future work.
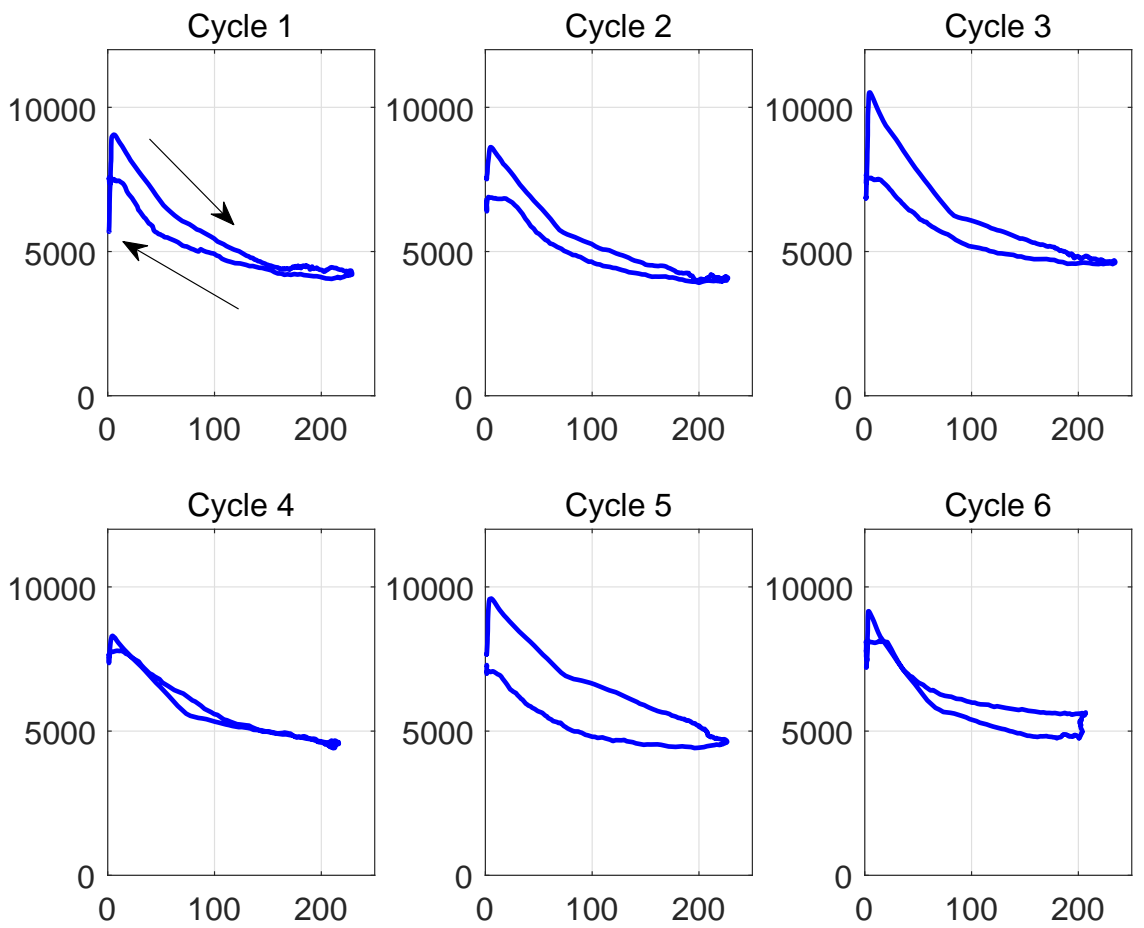

Figure 3: Downhole field data, torque [N.m] versus angular speed [RPM] at the bit: six stick-slip cycles available. The path from the top occurs when the bit accelerates, and the path from the bottom occurs when the bit speed decreases. The direction of the cycle is the same for all six cycles.

The mean cycle is obtained by applying a sliding window average, at each bit speed, for the upper phases (positive acceleration) and the lower phases (negative acceleration) separately. Figure 4 shows the field stick-slip cycles and their mean. The mean of all cycles (upper and lower) is in blue, and the mean cycle is shown in green. The upper green curve is the mean of the upper cycles and the lower green curve is the mean of the lower cycles. 


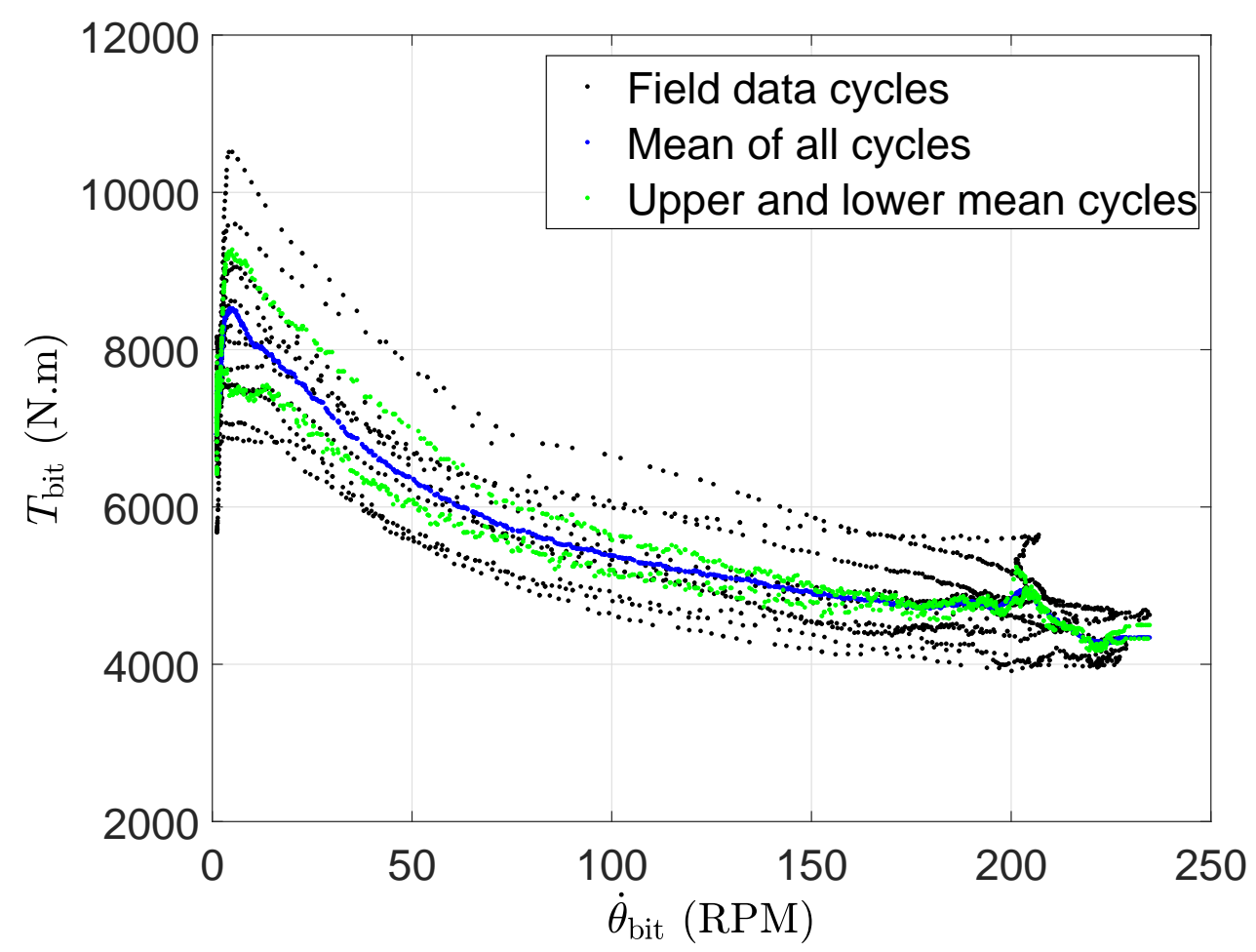

Figure 4: Downhole field data: six stick-slip cycles and their mean. The mean of all cycles (upper and lower) is in blue, and the mean cycle is shown in green.

If we take the green line shown in Fig. 4, i.e., the field data mean hysteretic cycle, it is remarked that the thickness of this cycle decreases as the bit speed increases. At some point, the upper and lower curves collapse.

A commonly used non-linear reversible bit/rock interaction model is given by $[4,11,15]$

$$
T_{\mathrm{bit}}\left(\dot{\theta}_{\mathrm{bit}}\right)=\mu \mathcal{W} \bar{r}\left(\tanh \left(b_{1} \dot{\theta}_{\mathrm{bit}}\right)+\frac{b_{2} \dot{\theta}_{\mathrm{bit}}}{1+b_{3} \dot{\theta}_{\mathrm{bit}}^{2}}\right),
$$

where $T_{\text {bit }}$ is the torque-on-bit in N.m, $\dot{\theta}_{\text {bit }}$ is the bit speed in $\mathrm{rad} / \mathrm{s}$. The parameters $b_{1}, b_{2}$, and $b_{3}$ depend on the bit and rock properties. The weighton-bit is $\mathcal{W}$, the friction coefficient is $\mu$, and the bit radius is $\bar{r}$. The weighton-bit, as expressed in the oil industry, is the amount of downward force exerted on the drill bit. 
Figure 5 shows separately the two terms of the reversible model, Eq. (11). The first term ( $\tanh$, in blue) increases fast, and reaches a limit torque value as the speed increases. The second term (fraction term, in blue) is responsible for the peak in the bit/rock interaction model, and it dies out as the bit speed increases. The reversible model, Eq. (11) is the sum of these two terms (magenta curve).

This model considers a steep straight line, when the bit speed is close to zero. Usually this strategy produces good results. One can find in the literature other models that take into account explicitly stick and slip phases, such as in [6].

Once fitted, the bit/rock interaction model described by Eq. (11) yields an acceptable agreement with the average experimental plots (blue plots in Fig. 4). Nevertheless it suffers from two drawbacks: (1) it is not flexible enough to fit correctly the experiments for both low and high velocities; and (2) it cannot generate hysteresis effects in order to separate the forward and backward behaviours (green plots in Fig. 4).

In order to improve the model shown in Eq. (11) we suggest two modifications to address these two issues: (1) the power exponents of the bit speed in the fraction term can vary in order to give the model more flexibility; and (2) the fraction term is modulated by an acceleration (and speed)-dependent factor to obtain different amplitudes for the forward and the backward phases.

The new bit/rock interaction model, proposed in the present paper and which contains these improvements, is presented in Eq. (12).

$$
T_{\text {bit }}\left(\dot{\theta}_{\mathrm{bit}}, \ddot{\theta}_{\mathrm{bit}}\right)=b_{0}\left(\tanh \left(b_{1} \dot{\theta}_{\mathrm{bit}}\right)+\frac{b_{2}\left|\dot{\theta}_{\mathrm{bit}}\right|^{b_{4}} \operatorname{sign}\left(\dot{\theta}_{\mathrm{bit}}\right)}{1+b_{3}\left|\dot{\theta}_{\mathrm{bit}}\right|^{b_{5}}}\left(1+H\left(\dot{\theta}_{\mathrm{bit}}, \ddot{\theta}_{\mathrm{bit}}\right)\right)\right),
$$

for $\dot{\theta}_{\text {bit }}>0$. It is usually observed that the reaction torque is much lower when the bit speed is negative. This is because the bit/cutters are not symmetrical. Actually, we do not have field data to support any interaction model for $\dot{\theta}_{\text {bit }}<0$, but, in the cases analyzed in the present paper, the values of the bit speed were always equal or greater than zero.

Note that if $b_{4}=1, b_{5}=2$ and $H=0$ we retrieve the bit/rock interaction model found in Eq. (11). The parameters $b_{4}$ and $b_{5}$, with $0<b_{4}<b_{5}$, depend on the bit and rock properties. The parameter $b_{0}$ [N.m] represents the weight-on-bit, the friction coefficient and the bit radius (for instance, this parameter might be written as $\left.b_{0}=\mu \mathcal{W} \bar{r}\right)$. 
The proposed model, Eq. (12), allows a better fit with the field data points. $H$ is the hysteretic function: if it is equal to zero, the model is reversible, with no hysteretic cycles. The hysteretic model is only activated if $H$ is different from zero, where $H$ is presented in Eq. (13).

$$
H\left(\dot{\theta}_{\mathrm{bit}}, \ddot{\theta}_{\mathrm{bit}}\right)=\beta_{1} \tanh \left(\beta_{2} \ddot{\theta}_{\mathrm{bit}}\right) \operatorname{sign}\left(\dot{\theta}_{\mathrm{bit}}\right),
$$

which means that the variation of the bit speed (bit angular acceleration) should be taken into account, and the hysteretic cycle is limited $\left(1 \pm \beta_{1}\right)$. Figure 6 shows the hysteretic term as a function of the bit acceleration. It increases fast, and reaches a limit value as the acceleration increases/decreases. In the present case $\beta_{1}=14 \%$, i.e., the hysteretic cycle is within plus or minus $14 \%$ of the reversible model. In addition, note that $(1-H)$ is multiplying only the second term of the bit/rock interaction model (the fraction term), such that as the bit speed increases the thickness of the hysteretic cycle decreases, as it is observed in most field data cycles shown in Fig. 3, and also in the field data mean cycle shown in Fig. 4. 


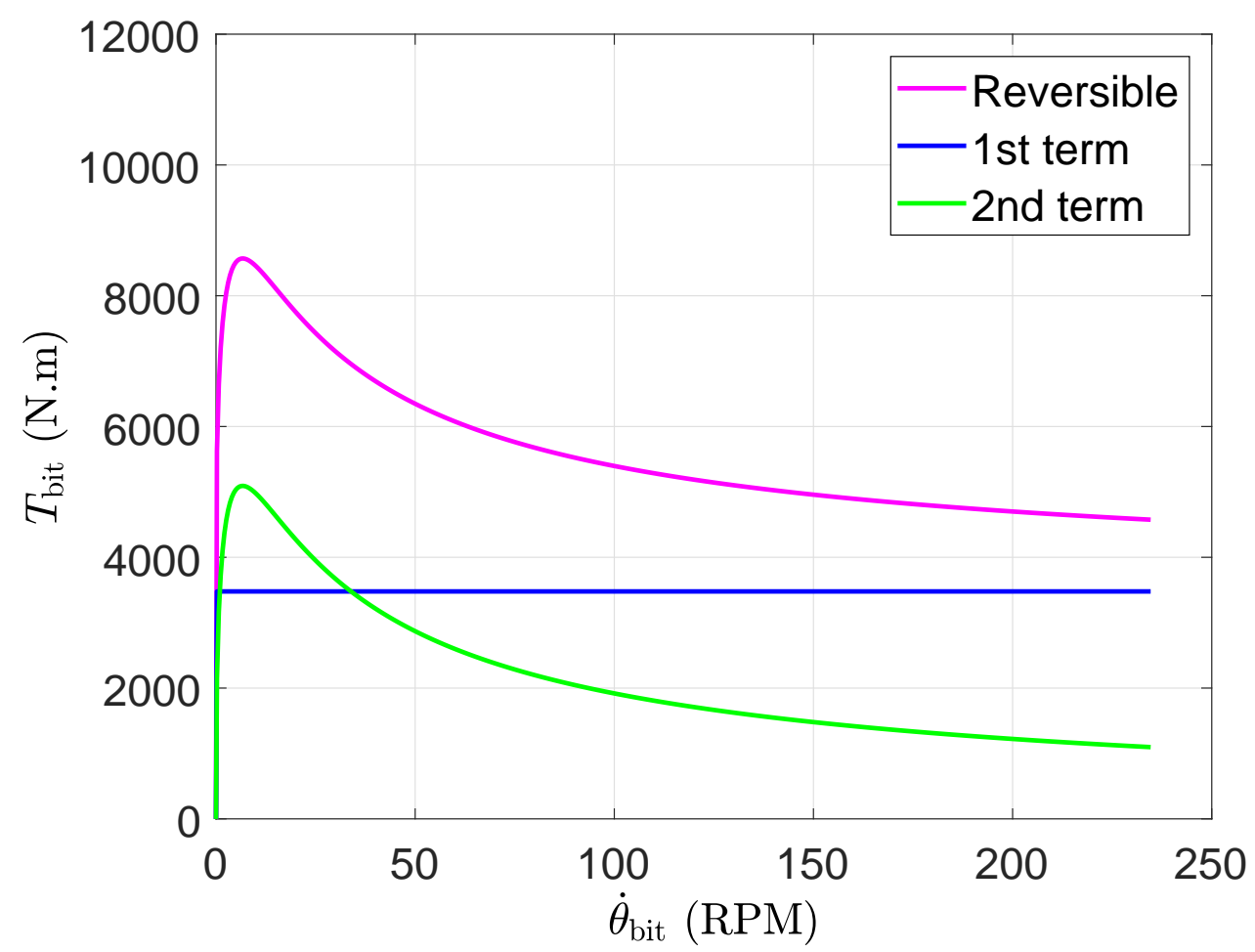

Figure 5: Terms of the bit/rock interaction reversible model, Eq. (11): tanh term in green, fraction term in blue, and complete reversible model in magenta. 

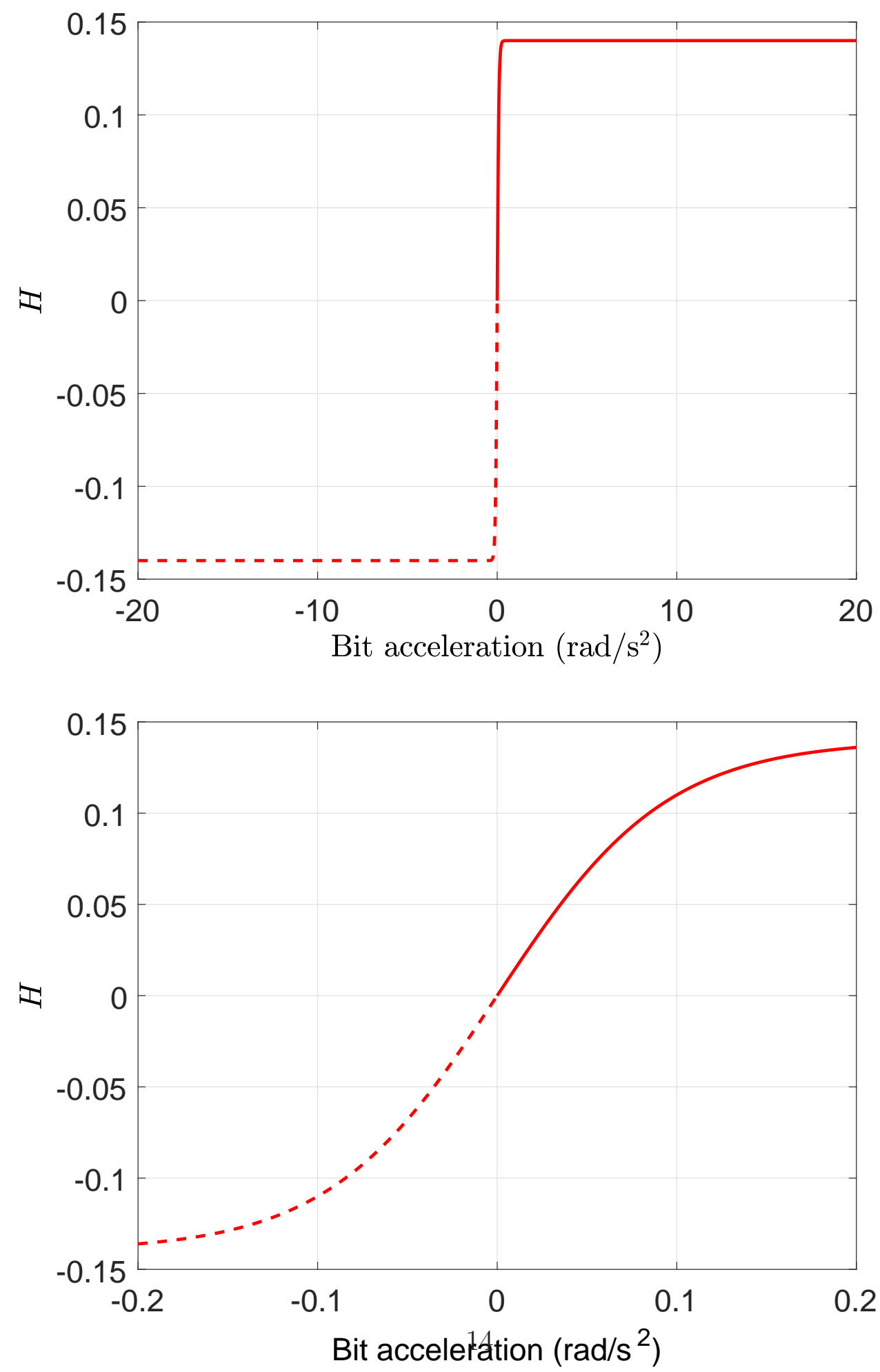

Figure 6: (a) Hysteretic term $H$ as a function of the bit acceleration and (b) zoom image. 
Figure 7 shows the hysteretic bit/rock interaction model for positive bit speeds. The arrows indicate the path of the cycle. The curve in blue happens when the bit acceleration is positive, and the curve in green happens when the bit accelaration is negative.

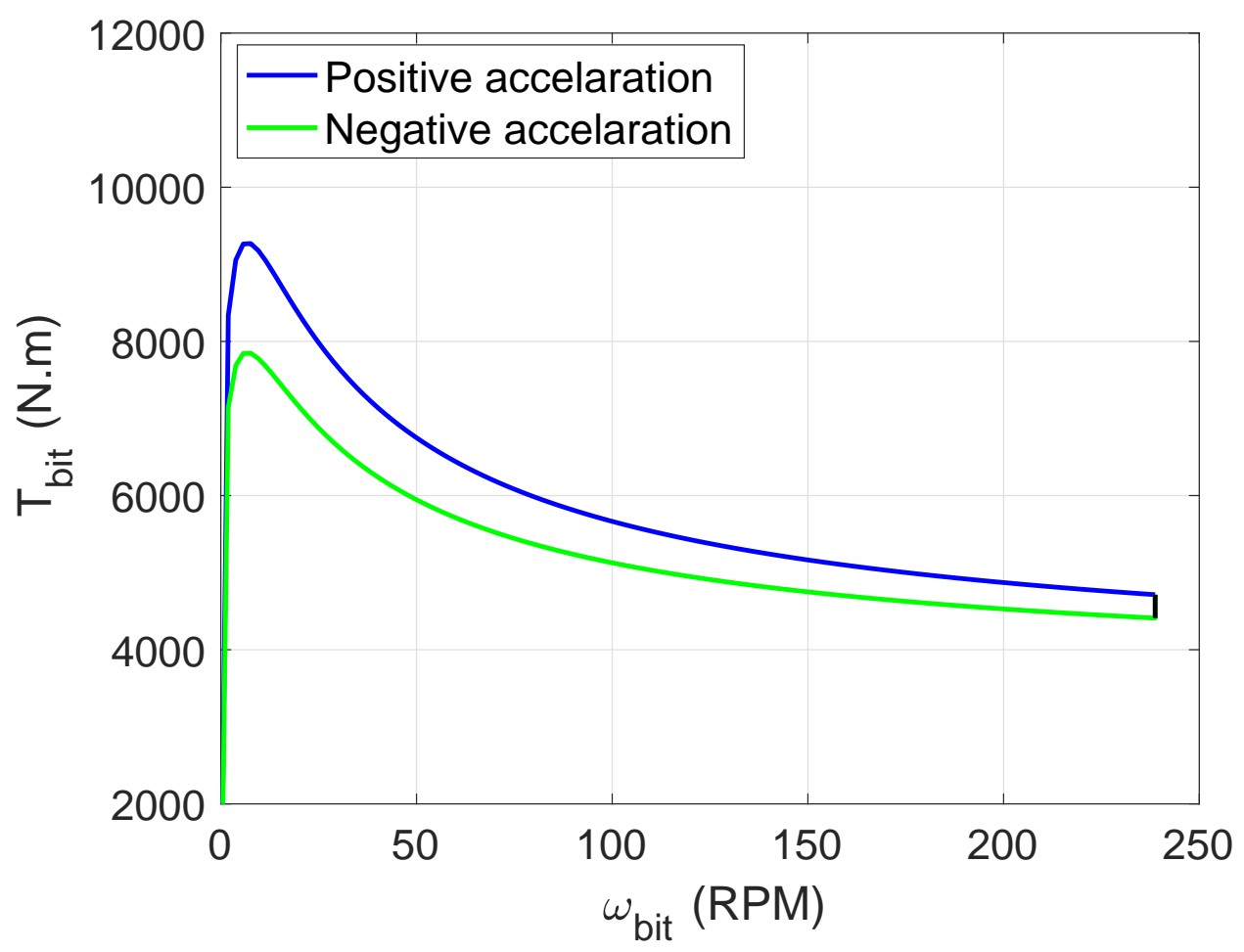

Figure 7: Hysteretic bit/rock interaction model. Blue line when the bit acceleration is positive and green line when the bit accelaration is negative. The black straight vertical line indicates when the acceleration switches from positive to negative values.

\section{Bit/Rock interaction: proposed model vs. field data}

First the reversible model is considered, Eq.12 with $H=0$. The fitted parameters of the bit/rock interaction model are: $b_{0}=3478, b_{1}=938$, $b_{2}=2.56, b_{3}=0.38, b_{4}=0.78$, and $b_{5}=1.1$, with appropriate units. Figure 8 shows the mean of the field data together with the fitted model. The blue line is the same field data blue line as shown in Fig. 4. There is a very good agreement between the bit/rock interection model and the field data. 


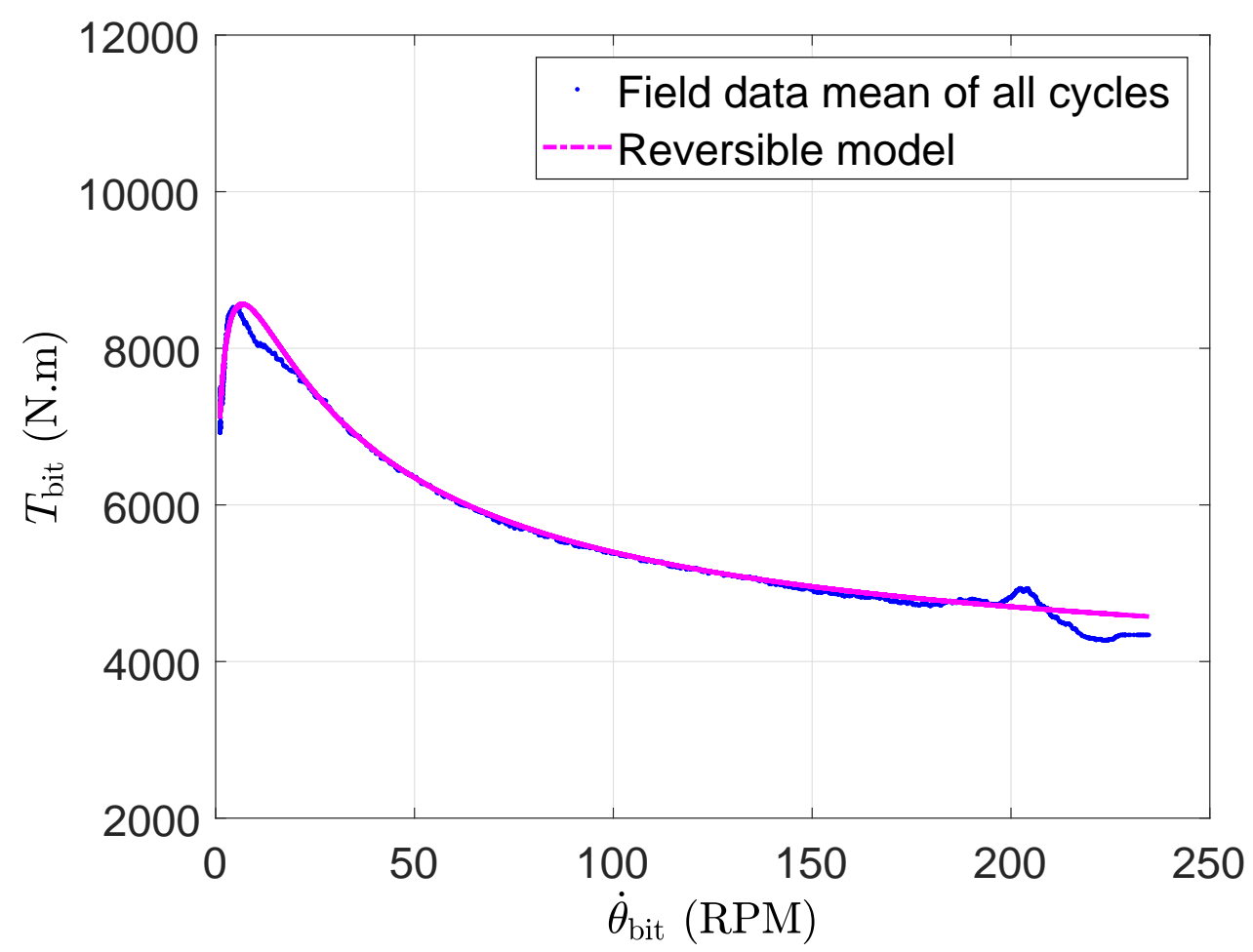

Figure 8: Field data vs. bit/rock interaction reversible model, (Eq. 11): Fitted model in magenta and mean field data in blue.

Now the model including hysteresis is considered, Eq.12 with $H \neq 0$. The fitted parameters of the hysteretic function are: $\beta_{1}=14 \%, \beta_{2}=10.6$, with appropriate units. Figure 9 shows the field data upper and lower mean cycles together with the fitted hysteretic bit/rock interaction model. The green lines are the same field data green lines as shown in Fig. 4. Again, there is a very good agreement between the proposed bit/rock interaction model and the field data. 


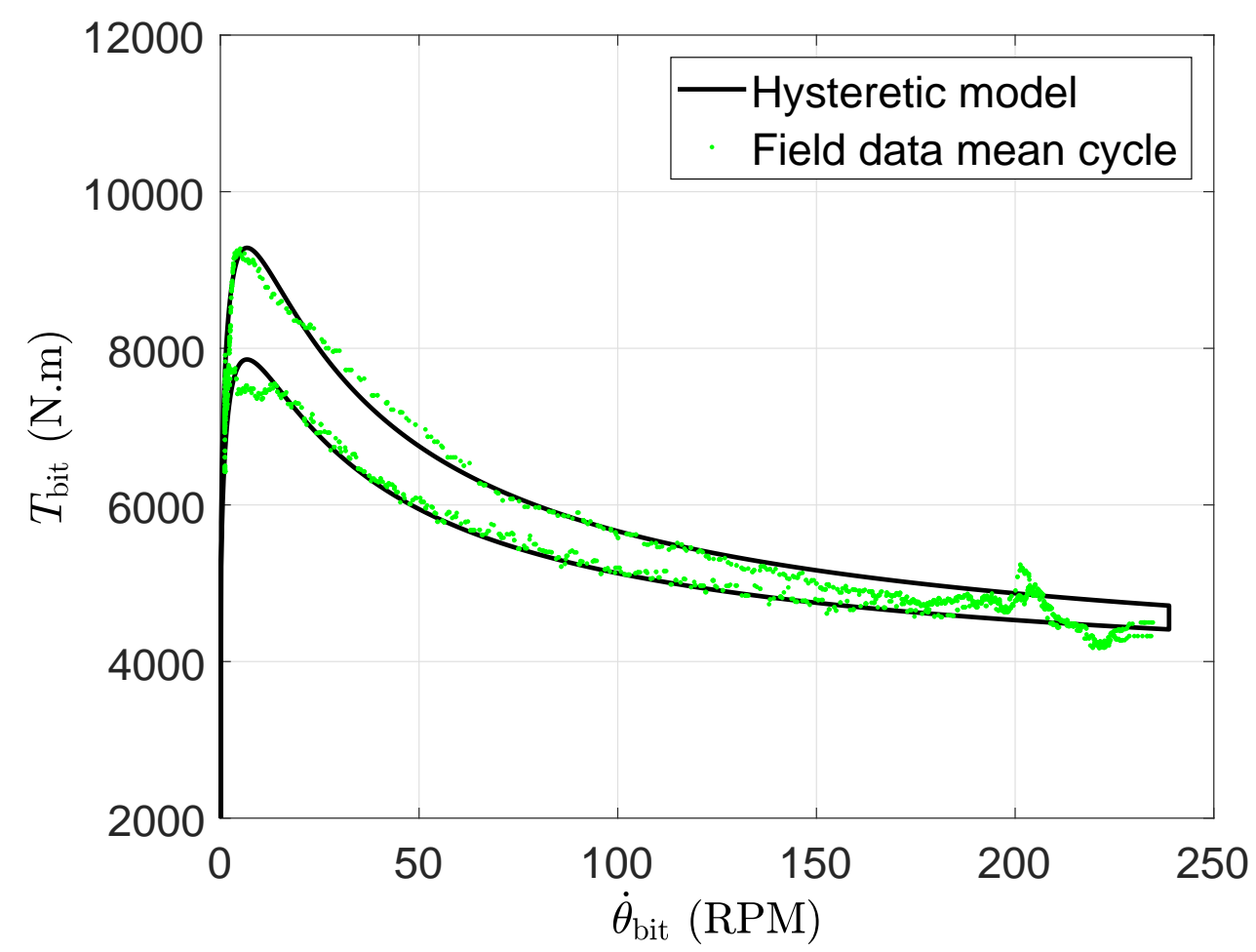

Figure 9: Field data vs. hysteretic bit/rock interaction model (Eq. 13): fitted model in black, and mean cycle of field data in green.

A larger hysteretic cycle is also considered, with $\beta_{1}=56 \%$. This parameter was chosen such that all field data cycles (black lines in in Fig. 4) fit in. Finally, Fig. 10 shows the three bit/rock interaction models that will be used for computations: (1) fitted reversible model (magenta line), (2) fitted model with hysteresis effects (continuous black line), and (3) model with large hysteresis effects (dashed black line).

The parameters used in the computational model are the ones that were fitted with the available field data: $b_{0}=3478, b_{1}=938, b_{2}=2.56, b_{3}=0.38$, $b_{4}=0.78, b_{5}=1.1$ and $\beta_{2}=10.6$. For model number one $H=0$, for model number two $\beta_{1}=14 \%$, and for model number three $\beta_{1}=56 \%$. 


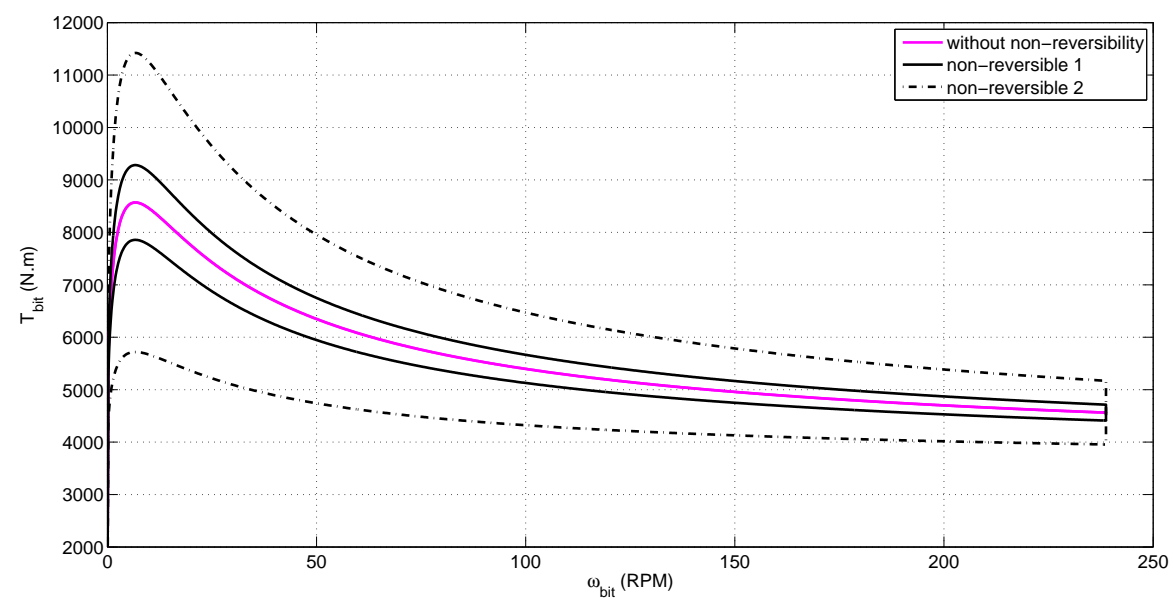

Figure 10: Bit/Rock interaction models (Eqs. 11 and 13): (1) reversible (magenta), (2) hysteretic $\beta_{1}=14 \%$ (black), and (3) hysteretic $\beta_{1}=56 \%$ (black).

\section{Numerical results}

In this section the results obtained for the three bit/rock interaction models are analyzed (see Fig. 10): (1) reversible (magenta), (2) hysteretic $\beta_{1}=14 \%$ (black), and (3) hysteretic $\beta_{1}=56 \%$ (black). The column is discretized in 100 finite elements. An adaptive Euler scheme is implemented to approximate the solution of the ODE. It is easy to implement and it is useful to compute the acceleration at each time step (which is necessary for the hysteretic $H$ function). The time step used $\Delta t=5 \times 10^{-4} \mathrm{~s}$ and each time step of the explicit integration scheme is computed as follows:

1. $\dot{\mathbf{q}}_{i+1}=\dot{\mathbf{q}}_{i}+\Delta t[\widetilde{M}]^{-1}\left(\tilde{\mathbf{T}}\left(\dot{\mathbf{q}}_{i}, \ddot{\mathbf{q}}_{i}\right)-[\widetilde{D}] \dot{\mathbf{q}}_{i}-[\widetilde{K}] \mathbf{q}_{i}\right)$

2. $\mathbf{q}_{i+1}=\mathbf{q}_{i}+\Delta t \dot{\mathbf{q}}_{i}$

3. $\ddot{\mathbf{q}}_{i+1}=\left(\dot{\mathbf{q}}_{i+1}-\dot{\mathbf{q}}_{i}\right) / \Delta t$

The values of the parameters of the drill string system analyzed in the present paper are the following ones. Material properties $\rho=7800 \mathrm{~kg} / \mathrm{m}^{3}$, $E=220 \mathrm{GPa}, \nu=0.29, G=E /(2(1+\nu))$, length of the drill pipe 4733.60 $\mathrm{m}$, outer and inner diameter of the drill pipe $0.140 \mathrm{~m}$ and $0.119 \mathrm{~m}$, length 
of the BHA $466.45 \mathrm{~m}$, outer and inner diameter of the BHA $0.1607 \mathrm{~m}$ and $0.0727 \mathrm{~m}$. The first five natural frequencies of the fixed-free torsional system, obtained using the stiffness and mass matrices of the finite element model, are $[0.1316,0.4199,0.7388,1.0719,1.4113] \mathrm{Hz}$, and the damping rates related to the first five normal models are $[0.095,0.02,0.02,0.02,0.02]$ (all the remaining damping rates are set to 0.02 ).

Figure 11 shows the bit speed time evolution when the nominal surface speed equals to $120 \mathrm{RPM}$ and $\mathcal{W}$ equals to $245 \mathrm{kN}$. The torsional oscillations are larger when employing the hysteretic bit/rock interaction model. All of the responses show stick-slip oscillations, where the bit speed is zero and the system accumulates strain energy up to the point that the bit speed is released and reaches more than two times the nominal surface speed. The frequency of stick-slip is around $0.128 \mathrm{~Hz}$ for model 1 (reversible), $0.126 \mathrm{~Hz}$ for model 2 (small reversibility), and $0.112 \mathrm{~Hz}$ for model 3 (large hysteresis effects). Hence, the stick-slip frequency is a little lower than the first torsional natural frequency of the system, and it decreases as the hysteresis of the bit/rock interaction increases.

In order to construct a stability map, the nominal surface speed is analyzed from 50 to 160 RPM, and the weight-on-bit from 5 to 60 klf (22 to $267 \mathrm{kN}$ ). The bit/rock interaction model shown in Eq. 12 was calibrated considering $245 \mathrm{kN}$, and the model assumes that $T_{\text {bit }}$ is linear with respect to the weight-on-bit, Eqs. (12) and (13). Thus, a coefficient is used to multiply $T_{\text {bit }}$ such that different values of $\mathcal{W}$ can be simulated. For example, if the $\mathcal{W}$ is $200 \mathrm{kN}$, then $T_{\text {bit }}$ must be multiplied by $200 / 245=0.816$. 


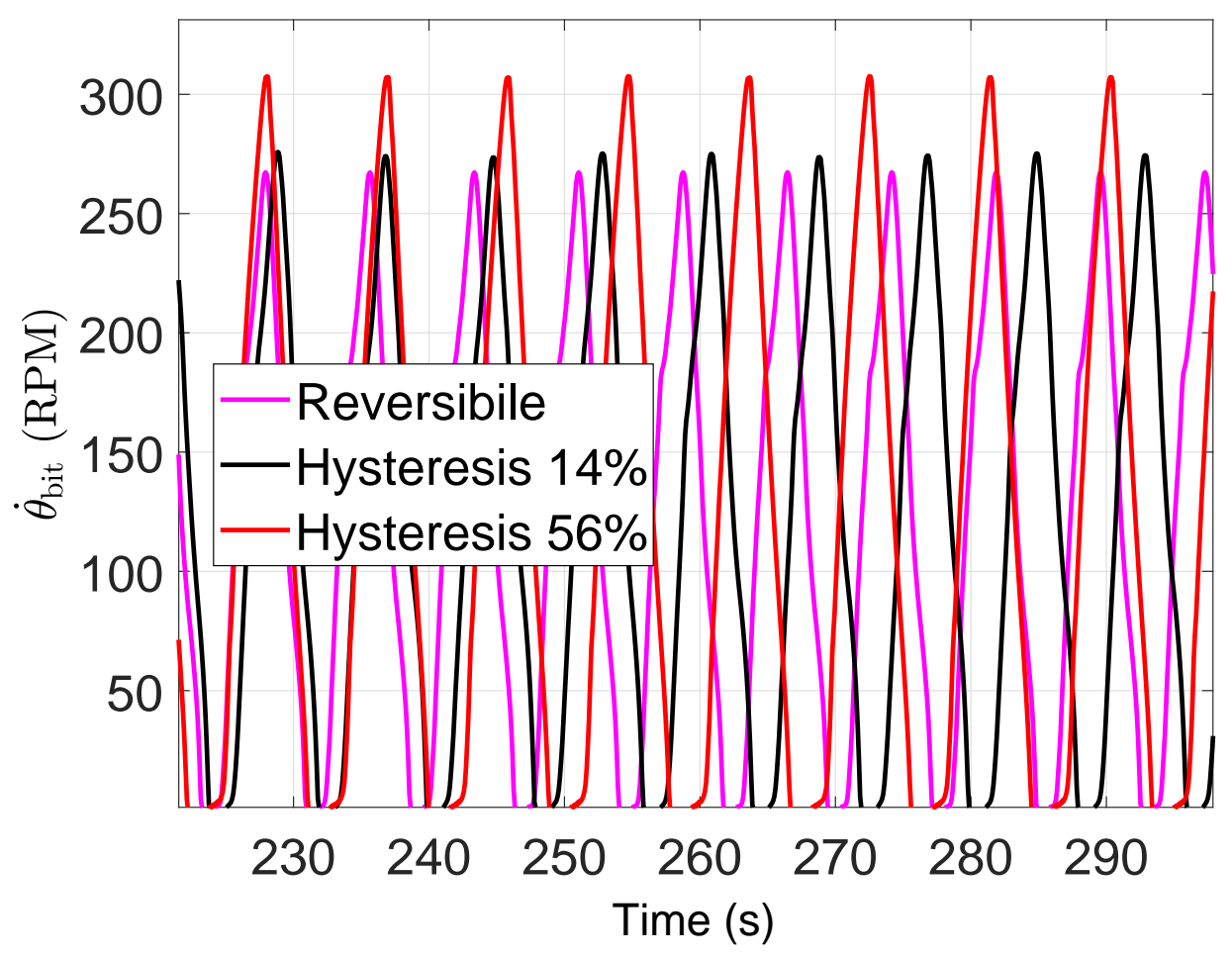

Figure 11: Bit speed for the three models (120 RPM, $245 \mathrm{kN})$ : (1) reversible (magenta), (2) hysteretic $\beta_{1}=14 \%$ (black), and (3) hysteretic $\beta_{1}=56 \%$ (red).

Let us define the stick-slip severity factor as $S S=\left(\omega_{\mathrm{bit}}^{\max }-\omega_{\mathrm{bit}}^{\min }\right) /(2 \Omega)$. If there is no torsional oscillations, $S S=0$. If there is stick-slip oscillations $\left(\omega_{\mathrm{bit}}^{\min }=0\right)$ and the maximum bit speed $\left(\omega_{\mathrm{bit}}^{\max }\right)$ is two times the nominal surface speed $(\Omega)$, then $S S=1$. If $S S$ is lower than 0.5 the system will be considered stable, otherwise it is considered unstable.

The stability map is constructed as follows. For each pair $(\mathcal{W}, \Omega)$ the bit speed is computed and $S S$ is recorded for the steady state response. Figure 12 shows the stability map when employing the bit/rock interaction models 1 (reversible) and 2 (small hysteresis effects). It can be noted that the maximum $S S$ value is greater for the reversible bit/rock interaction model (model 2), but, at the same time, the stability region (dark blue) is a little bigger when employing this model. Now let us analyze Fig. 13, which shows the stability map when employing the bit/rock interaction model 3 (large hysteresis effects). This last chart presents a stability region (dark blue 
region) bigger than the other two charts.

This means, on one hand, that the hysteresis in the bit/rock interaction model favours the stability of the system. It seems that when the bit speed decreases, and $T_{\text {bit }}$ is in the lower curve of the hysteretic cycle (see Fig. 10), it allows the system to escape from instability. On the other hand, it can also be obverved that the instability region (orange-red region) in Fig. 13 presents values of $S S$ higher than the charts in Fig. 12. Hence, at the same time that the hysteresis in the bit/rock interaction model favours the stability of the system for low values of weight-on-bit and high values of nominal surface speed, it favours the aggravation of the torsional oscillations of the system for high values of weight-on-bit and low values of nominal surface speeds. Since $T_{\text {bit }}$ passes through the upper curve of the hysteretic cycle (see Fig. 10) the amplitude of the stick-slip oscillations increases as well. 

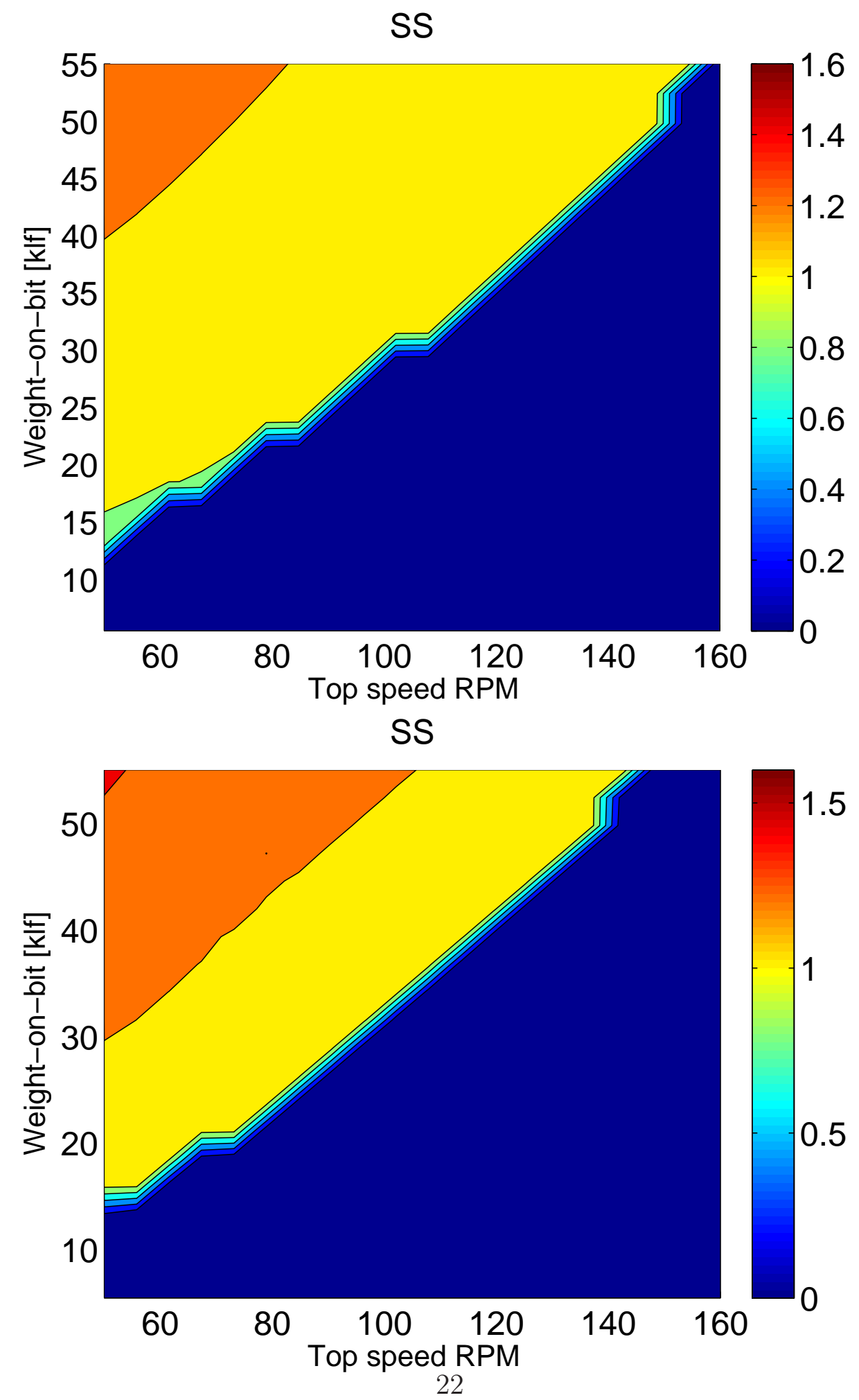

Figure 12: Stability map for models 1 (reversible) and 2 (hysteretic $\beta_{1}=14 \%$ ). 


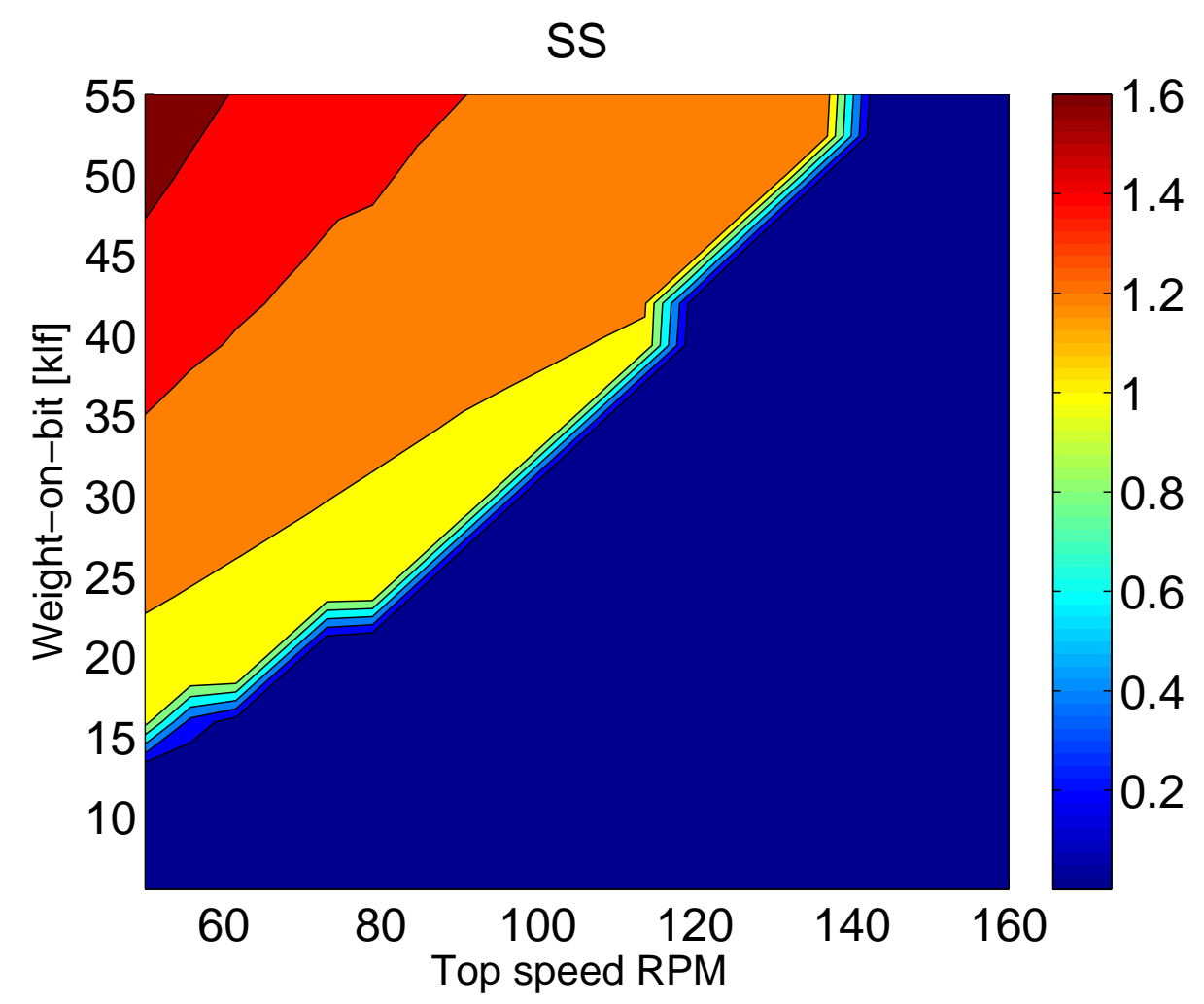

Figure 13: Stability map for the model 3 (hysteretic $\beta_{1}=56 \%$ ).

\section{Concluding Remarks}

The present paper proposes a bit/rock interaction model including a type of hysteresis (non-reversibility). The proposed model is based on field data and a hysteretic model is applied to represent the bit/rock interaction model of a drill string system.

Only torsional vibrations are analyzed for a torsion bar system discretized by means of the finite element method. A reduced-order model was constructed to speed up the computations. The torsional dynamics of the system is analyzed, and the stability map shows that the system including a bit/rock interaction model with hysteresis effects (1) favours the stability of the system for high nominal surface speeds and low weight-on-bit, and (2) induces higher stick-slip oscillations for low nominal surface speeds and high weight-on-bit. The reason for that has to do with the hysteretic cycle of the torque-on-bit versus the bit speed. 
Since this paper presents the first results obtained employing this model, more investigations must be made with the proposed hysteretic bit/rock interaction model. In addition, the authors are now working on a stochastic model to take into account the fluctuations of the hysteretic cycles, which are present in the field data.

\section{Acknowledgments}

The fourth author would like to acknowledgment the finantial support of the Brazilian agencies CNPq, CAPES, and FAPERJ. The authors would also like to acknowledge Schlumberger Oilfield Services for publishing this article.

\section{References}

\section{References}

[1] L. Hong, I. P. Girsang, J. S. Dhupia, Identification and control of stick?slip vibrations using Kalman estimator in oil-well drillstrings, Journal of Petroleum Science and Engineering, 140 (2016) 119?127.

[2] D. Inman, Engineering Vibration, Pearson, 4th edition, (2013).

[3] G. Kerschen, J.-C. Golinval, A. F. Vakakis, L. A.Bergman, The method of proper orthogonal decomposition for dynamical characterization and order reduction of mechanical systems: An overview, Nonlinear Dynamics 41(1-3) 2005 147-169.

[4] Y. A. Khulief, F. A. Al-Sulaiman, S. Bashmal, Vibration analysis of drillstrings with self-excited stick-slip oscillations, Journal of Sound and Vibration, 299 (3) (2007) 540-558.

[5] E. Kreuzer, M. Steidl, Controlling torsional vibrations of drill strings via decomposition of traveling waves, Archive of Applied Mechanics, 82(4) (2017) 515-531.

[6] R. I. Leine, D. H. van Campen, W. J. G. Keultjes, Stick-slip Whirl Interaction in Drillstring Dynamics, Journal of Vibration and Acoustics, 124 (2002) 209-220. 
[7] N. Mihajlovic, N. Van De Wouw, M. P. M. Hendriks, H. Nijmeijer, Friction-induced limit cycling in flexible rotor systems: An experimental drill-string set-up, Nonlinear Dynamics 46 (3) (2006) 273-291.

[8] E.M. Navarro-López, E. Licéaga-Castro, Non-desired transitions and sliding-mode control of a multi-DOF mechanical system with stick-slip oscillations, Chaos, Solitons and Fractals 41 (4) (2009) 2035-2044.

[9] D. R. Pavone, J. P. Desplans, Application of high sampling rate downhole measurements for analysis and cure of stick-slip in drilling, Proceedings - SPE Annual Technical Conference and Exhibition Delta, (1994) 335-345.

[10] Patil, P.A., Teodoriu, C., A comparative review of modelling and controlling torsional vibrations and experimentation using laboratory setups, Journal of Petroleum Science and Engineering, 112 (2013) 227-238.

[11] M. T. Piovan, R. Sampaio, Continuous models for drill-strings of the oil industry: Analysis of approaches and discretization schemes, Revista Internacional de Metodos Numericos para Calculo y Diseno en Ingenieria, 25 (3) (2009) 259-277.

[12] T. Richard, C. Germay, E. Detournay, A simplified model to explore the root cause of stick-slip vibrations in drilling systems with drag bits, Journal of Sound and Vibration, 305(3) (2007) 432-456.

[13] T. G. Ritto, C. Soize, R. Sampaio, Non-linear dynamics of a drill-string with uncertain model of the bit-rock interaction, International Journal of Non-Linear Mechanics 44 (8) (2009) 865-876.

[14] T. G. Ritto, F. S. Buezas, R. Sampaio, A new measure of efficiency for model reduction: Application to a vibroimpact system, Journal of Sound and Vibration 330(9) (2011) 1977-1984.

[15] T. G. Ritto, R. Sampaio, Stochastic drill-string with uncertainty on the imposed speed and on the bit-rock parameters, International Journal for Uncertainty Quantification, 2 (2) (2012) 111-124.

[16] T. G. Ritto, M. R. Escalante, R., Sampaio, M.B. Rosales, Drill-string horizontal dynamics with uncertainty on the frictional force, Journal of Sound and Vibration, 332 (1) (2013) 145-153. 
[17] T. G. Ritto, Bayesian approach to identify the bit-rock interaction parameters of a drill-string dynamical model, Journal of the Brazilian Society of Mechanical Sciences and Engineering, 37 (4) (2015) 1173-1182.

[18] T. G. Ritto, R.R. Aguiar, S. Hbaieb, Validation of a drill string dynamical model and torsional stability, Meccanica, 52 (2017) 2959-2967.

[19] Y. Shen, Z. Zhang, J. Zhao, W. Chen, M. Hamzah, R. Harmer, and G. Downton, The Origin and Mechanism of Severe Stick-Slip, Society of Petroleum Engineers, SPE-187457-MS (2017).

[20] J. Shi, B. Durairajan, R. Harmer, W. Chen, F. Verano, Y. Arevalo, C. Douglas, T. Turner, D. Trahan, J. Touchet, Y. Shen, A. Zaheer, F. Pereda, K. Robichaux, D. Cisneros, Integrated Efforts to Understand and Solve Challenges in 26-in Salt Drilling, Gulf of Mexico. SPE 180349MS , SPE Deepwater Drilling \& Completions Conference, Galveston, Texas, USA (2016).

[21] R. W. Tucker, C. Wang, An integrated model for drill-string dynamics, Journal of Sound and Vibration, 224 (1) (1999) 123-165.

[22] R. W. Tucker, C, Wang, Torsional vibration control and cosserat dynamics of a drill-rig assembly, Meccanica, 38(1) (2003) 143-159.

[23] R. Viguié, G. Kerschen, J.-C. Golinval, D.M. McFarland, L.A. Bergman, A. F. Vakakis, N, van de Wouw, Using passive nonlinear targeted energy transfer to stabilize drill-string systems, Mechanical Systems and Signal Processing, 23 (1) (2009) 148-169.

[24] J. Wojewoda, A. Stefanski M. Wiercigroch, T. Kapitaniak, Hysteretic effects of dry friction: modelling and experimental studies, Philosophical Transactions of the Royal Society A, 366 (2008) 747-765.

[25] J. Woodhouse, T. Putelat, A. McKay, Are there reliable constitutive laws for dynamic friction?, Philosofical Transactions A, 373(20140401) (2015) DOI: http://dx.doi.org/10.1098/rsta.2014.0401. 